\title{
El Patronato Provincial de Menores: iniciativas por la infancia pobre en la provincia de Buenos Aires (1917-1921)
}

\section{The Provincial Children's Welfare Board: Initiatives for Poor Children in the Province of Buenos Aires (1917-1921)}

\author{
Yolanda de Paz Trueba* \\ (D) https://orcid.org/0000-0002-9011-7522 \\ Instituto de Estudios Históricos y Sociales \\ Instituto de Geografía, Historia y Ciencias Sociales \\ Universidad Nacional del Centro de la Provincia de Buenos Aires \\ Conicet, Argentina \\ yolidepaz@gmail.com
}

Resumen: Este trabajo parte de preguntarse si el advenimiento del radicalismo a la gobernación de la provincia de Buenos Aires tuvo influencia en la implementación de políticas por la infancia que, como el Patronato de Menores, eran largamente reclamadas por la opinión pública y habían sido motorizadas por los anteriores gobiernos conservadores, quienes sin embargo, por diversas contingencias, no pudieron llevarlas a cabo. Con la intención de aportar a la

* Doctora en Historia por la Universidad Nacional del Centro de la Provincia de Buenos Aires. Profesora adjunta de la carrera de Historia en la UNCPBA. Investigadora adjunta del CoNICET. Ha investigado sobre el lugar de las mujeres en la esfera pública y desarrolla proyectos sobre las intervenciones sobre la infancia entre 1880 y 1920 en la provincia de Buenos Aires.

cómo citar: De Paz Trueba, Y. (2020). El Patronato Provincial de Menores: iniciativas por la infancia pobre en la provincia de Buenos Aires (1917-1921). Secuencia (106), e1706. Dor: https://doi.org/10.18234/ secuencia.v0i106.1706

c) 98

Esta obra está protegida bajo una Licencia Creative Commons Atribución-NoComercial 4.0 Internacional. 
historia social de la infancia, se asume que el primer gobernador radical no lo tuvo entre sus prioridades en los primeros tiempos de su mandato.

Palabras clave: Patronato de Menores; infancia; radicalismo; políticas sociales.

Abstract: This article begins by asking whether the advent of radicalism in the governorship of the province of Buenos Aires influenced the implementation of policies for children which, like the Children's Board, had long been called for by public opinion and had been mobilized by previous conservative governments, which, however, due to various contingencies, had been unable to implement them. With the aim of contributing to the social history of childhood, it is assumed that the first radical governor did not prioritize them during the early stages of his administration.

Key words: Provincial Children's Welfare Board; childhood; radicalism; social politics.

Recibido: 10 de enero de 2019 Aceptado: 3 de abril de 2019

Publicado: 5 de diciembre de 2019

$\mathrm{E}$ n 1910, José Inocencio Arias llegó a la gobernación de la provincia de Buenos Aires promovido por su antecesor Ignacio D. Irigoyen y en representación del recientemente creado Partido Conservador. ${ }^{1} \mathrm{Su}$ mandato estuvo signado, entre otras cosas, por varios proyectos que, con poco éxito, buscaron centralizar el poder en la figura del gobernador en detrimento de los municipios, y por una época de aires reformistas que vio nacer innovaciones como la reforma electoral que consagró el sufragio secreto y obligatorio a nivel nacional en $1912 .^{2}$

1 Fue fundado en 1908 a partir de una reorganización que buscaba revitalizar el poder del oficialismo que bajo la denominación Partido Autonomista Nacional (PAN) había gobernado la nación durante las últimas cinco décadas.

${ }^{2}$ La provincia de Buenos Aires adecuó su sistema en 1913. Interpretaciones críticas analizan la reforma electoral desde un ángulo que relativiza la postura que la planteaba como pro- 
$\mathrm{Al}$ poco tiempo de llegado al poder, manifestó ciertas preocupaciones por la situación social de la infancia que tuvo como resultado la sanción en 1910 de la Ley de Patronato de Menores de la provincia de Buenos Aires, que preveía la creación de una institución modelo destinada a dar albergue y formar para el trabajo a la mayor cantidad de niños y niñas posible y que por diversas razones estaban a cargo de los defensores de menores. La promoción de una institución como esta respondía a la convicción de que era necesario impulsar una manera más moderna de atender a estos problemas, en sintonía con la intención de centralizar políticas en manos provinciales restándole poder a los mandatarios locales. ${ }^{3}$ El gobierno conservador de Arias trabajó para hacer realidad la sanción de la ley que lo instituía, pero no logró llevarlo a la práctica; el gobernador falleció en el ejercicio de su cargo en 1912 antes de que el Patronato fuera un hecho. El difícil contexto que tuvieron que atravesar los gobiernos conservadores que lo sucedieron en materia económica, sumados a las luchas políticas y a la escisión irreconciliable entre facciones internas del partido gobernante, pusieron a algunas obras públicas como la que es objeto de estudio aquí, en una larga lista de espera. Como he sostenido para un periodo previo (De Paz Trueba, 2018b), las urgencias económicas, pero especialmente los vaivenes de la política, ganaron la batalla de las prioridades en las agendas gubernamentales, poco dispuestas en los hechos (salvo excepciones), a ocuparse de los problemas sociales de la infancia.

El 24 de abril de 1917, el presidente radical Hipólito Yrigoyen decretó la intervención federal de la provincia de Buenos Aires, designando al frente de la misma a José Luis Cantilo. La intención manifiesta de esta acción era sanear las instituciones erosionadas por las políticas caudillistas y el fraude electoral instauradas por los gobiernos de signo conservador. Un objetivo menos pregonado, aunque no por ello menos evidente, era quitar a estos el control de la provincia de Buenos Aires, crucial para Yrigoyen de cara a acrecentar su poder y controlar el principal bastión electoral. ${ }^{4}$

ducto de la modernización política impulsada por sectores que buscaban la democratización del sistema (Tato, 2005); Fernández Irusta, 2009), entre otros.

${ }^{3}$ La sanción de la ley no se puede aislar de su programa más general y de la cuestión de la disputa por las autonomías municipales. Sobre la relación entre estas y la cuestión de la infancia, véase De Paz Trueba (2018a).

${ }^{4}$ Con esta medida, llegaba a su fin el dominio del Partido Autonomista Nacional que con distintas denominaciones (y sólo un breve interregno entre 1894 y 1902 con las gobernaciones de Guillermo Udaondo por la Unión Cívica Nacional y Bernardo de Irigoyen por el radicalismo), había gobernado la provincia entre 1880 y 1917. Así, el interventor José Luis Cantilo 
Este trabajo (que se ubica entre 1917 -año que marcó el fin del predominio conservador en la provincia de Buenos Aires-y 1921 -cuando finalizó la gestión de José Camilo Crotto, primer gobernador radical electo por este partido bajo la nueva legislación electoral-), parte de preguntarse si este cambio de signo político tuvo influencia en la implementación de políticas por la infancia como el Patronato.

En junio de 1920, a poco de irse del gobierno, Crotto puso la piedra fundamental del que sería el edificio definitivo de la institución que por entonces funcionaba en un local provisorio que no reunía las condiciones necesarias para hacer realidad el proyecto que la ley preveía. ${ }^{5}$ ¿Debe hacernos pensar esto en un mayor compromiso hacia la cuestión social por parte de los radicales?, ¿es preciso por el contrario asumir que otras prioridades retrasaron la ejecución de las obras? Una respuesta lineal no daría cuenta de la complejidad del proceso. Si bien algunos hombres del radicalismo mostraron especial preocupación por el tema, como se verá en las páginas siguientes, no lo hicieron en mayor medida que sus contemporáneos socialistas y aun los conservadores con quienes en términos generales los separaban más cuestiones electorales que doctrinarias.

La historiografía política demostró hace tiempo que hay que ser cautos a la hora de interpretar la llegada del radicalismo al poder como un parteaguas, como un momento de ruptura en el que las promesas y reclamos de modernización y claridad del juego político se hicieron por fin realidad (Ferrari, 2008; Hora, 2013; Persello, 2004). La misma ley electoral que consagró el sufragio electoral libre, secreto y obligatorio a nivel nacional, no garantizó en términos generales que eso impulsara efectivamente una mayor transparencia, a pesar de que sí permitió desde entonces incorporar nuevos actores al mismo. En el caso de la provincia de Buenos Aires en particular, diversos historiadores han señalado no obstante de qué manera y a través de qué artilugios legales la clientela política dio paso a prácticas de partido y de comité (Béjar, 2002; Castro, 2000; Fernández Irusta, 2006; Ferrari, 2012; Melón

se enfrentó a la tarea de reorganizar los poderes públicos y la administración de la provincia en el intento de desarmar la estructura montada por el anterior gobierno de Marcelino Ugarte, orientada a mantener el control político de la provincia.

${ }^{5}$ Funcionó por un tiempo en un local de la calle 6 entre 58 y 59 de la ciudad de La Plata, capital provincial. Allí, se albergaban poco más de 60 menores, todos de sexo masculino. Las reducidas dimensiones del lugar no sólo no permitían dar socorro a la cantidad de chicos que la ley estipulaba. Tampoco era posible recoger a las menores de sexo femenino, además de que no contaba con espacio suficiente para desarrollar los talleres que constituían la piedra angular del proyecto pensado para asilar, pero sobre todo para formar. 
Pirro, 1994; Tato, 2005). Además, otros trabajos han pensado para el mismo periodo las dificultades económicas y el agravamiento de las tensiones en medio de las que los diversos gobiernos debieron hacer frente al temporal, y llevar adelante gestiones en un contexto menos halagüeño que sus antecesores (Palacio, 2000; Rocchi, 2013). La historiografía de la infancia, por su parte, ha estudiado la sanción de la Ley de Patronato de Menores 10.903 de 1919 (que tuvo injerencia a nivel de la Capital Federal y los territorios nacionales) como un logro conseguido en ese mismo marco y en parte relacionada con la agudización de las problemáticas sociales que se dio tanto a nivel nacional como provincial (Zapiola, 2014). ${ }^{6}$ Autores que reflexionan sobre otras situaciones provinciales encuentran también una cierta correlación entre el advenimiento del radicalismo y el desarrollo de una incipiente política social que habría tenido mayor desarrollo con la llegada del peronismo (Cerdá, 2013).

Esos abordajes han estimulado en gran parte las reflexiones de este trabajo, y nos han llevado a preguntarnos por el lugar que debemos darle, en un momento de insistentes reclamos retóricos y reformas políticas menos rupturistas, a los avances y demoras en materia de políticas por la infancia en la provincia de Buenos Aires. La prensa de diversos signos políticos, las discusiones en las cámaras legislativas, el Registro Oficial de la provincia, y demás documentación estatal, serán puertas de acceso que permitirán dar cuenta de las cuestiones que nos ocupan.

${ }^{6}$ Cabe señalar que existía un trasfondo de preocupaciones en torno a la infancia en el periodo analizado que no se circunscribía de manera exclusiva a la provincia de Buenos Aires, sino que estaba presente también en otras provincias y aun en el ámbito nacional. De hecho, y tal como ha sostenido Carolina Zapiola (2014), la primera presentación del proyecto de Ley de Patronato de Menores (la mencionada Ley 10903) al Congreso Nacional por parte del diputado Luis Agote y su discusión se realizó también en 1910. Sin embargo, y al margen de que la ley provincial fuera efectivamente sancionada en ese año (aunque su implementación fuera mucho más lenta) y la nacional recién llegara a concretarse en 1919, debemos señalar que se trató de dos cuerpos legales de distinta naturaleza. Mientras la provincial pretendía solucionar el problema de la infancia abandonada a través de una institución que pretendía recoger y asilar a los niños que quedaban bajo la órbita de las Defensorías de Menores, conteniendo un propósito de prevención para evitar la caída de este colectivo en la delincuencia, la ley nacional instauró por primera vez una legislación penal específica para el tratamiento de menores delincuentes. Ambas leyes vieron su origen en el marco de preocupaciones similares (cómo evitar que los chicos que pasaban largas horas en las calles cayeran en la delincuencia y, de hacerlo, juzgarlos y contar con espacios específicos para su tratamiento). No obstante, durante la discusión de la ley en la legislatura bonaerense, no se hicieron menciones específicas al proyecto de Agote. En cambio las observaciones al proyecto original estuvieron centradas en la manera en que se llevaría a cabo su financiamiento, a la sazón un asilo de grandes dimensiones destinado a chicos y chicas de toda la provincia. 
Considero, entonces, que los avances de la etapa radical obedecieron a un conjunto de motivos donde se mixturaron la urgencia social de actuar por la infancia pobre y las presiones políticas y de la opinión pública, ${ }^{7}$ que pusieron al gobierno provincial ante la obligación de retomar, aunque de manera inconclusa, un propósito que hundía sus raíces en el periodo anterior. En definitiva, el nuevo contexto político y social, aunque no el económico, fue propicio para dar principio a un proyecto que había sido definido en sus formas, alcances y contenido por los conservadores, y que el primer gobernador radical no tuvo entre sus prioridades en los primeros tiempos de su gobierno. ${ }^{8}$

\section{CRISIS ECONÓMICA Y CUESTIONES SOCIALES: PROBLEMAS Y SOLUCIONES NUEVAS}

En 1917, el mundo estaba sacudido por una contienda bélica que involucraba a las naciones más importantes del mundo. Argentina, cuyo crecimiento en gran parte se había dado a raíz de su conexión con los mercados mundiales, no permaneció ajena a las consecuencias que esto acarreó.

Principalmente se vieron afectados los flujos de capitales y el comercio con el exterior, en los que se basaba la economía argentina y su naciente industria, que desde 1870 se había comenzado a desarrollar en la ciudad de Buenos Aires, primero, aumentando considerablemente la participación de la provincia, después. Para 1914 esta contaba con casi 15000 establecimientos industriales, lo que representaba 30\% del número total del país (Rocchi, 2013, p. 94). La producción se apoyaba sobre todo en insumos importados, y con la guerra se cortaron los flujos de materia prima, lo que afectó severamente a la industria. Desde 1914 hasta 1918 el impacto de la guerra en el comercio internacional y en las finanzas argentinas contribuyó a la reducción de los puestos de trabajo ( $20 \%$ en el país) y la caída del valor adquisitivo de los salarios fue grave (Ascolani, 2013, pp. 404-405). La joven industria retomaría recién su rit-

${ }^{7}$ Entiendo por opinión pública las apreciaciones generales que sobre los temas aludidos manifestaba la sociedad en general y algunos individuos (intelectuales y políticos) en particular a través de la prensa, las discusiones legislativas y otros documentos oficiales que se usan en este trabajo.

${ }^{8}$ Cabe subrayar que este trabajo no pretende dar cuenta de un compromiso mayor o menor del radicalismo hacia la implementación de políticas sociales, sino solamente se centra en un aspecto de la misma (las intervenciones en favor de la infancia). 
mo de crecimiento luego de 1920 (Rocchi, 2013). No obstante, como sostiene Juan Manuel Palacio (2000), "el periodo que inaugura la guerra es claramente uno de transición entre una época y otra, que a la vez que sigue viviendo del crecimiento hacia afuera convive también con los elementos del mundo que viene". En él, entre otras cosas, se observa un incremento de la participación de la industria en el producto bruto nacional, el estancamiento de las inversiones británicas y un mayor protagonismo de Estados Unidos, una reorientación del comercio exterior, y una pérdida de importancia del ferrocarril que comenzó a sufrir la competencia de los automóviles y las carreteras (Palacio, 2000 , p. 105). En síntesis, ya nada volvería a ser como antes aunque se pueden identificar periodos de expansión una vez pasado el momento más crítico.

Así, en medio de una situación compleja en materia económica, las páginas de la prensa estaban plagadas de notas que daban cuenta de estas dificultades y de una de sus consecuencias más inmediatas y visibles: el aumento de la mendicidad callejera y el vagabundaje, especialmente de los menores de edad, muchos de los cuales, según decía El Día de la ciudad de la Plata, eran hijos de los obreros que se agolpaban a las puertas de los frigoríficos y fábricas buscando un trabajo que no tenían. ${ }^{9}$

Esta situación no sólo afectaba a las grandes ciudades de la provincia. Las más pequeñas del interior también sufrieron el impacto, puesto que, como señalaba El Ciudadano de la ciudad de Azul ${ }^{10}$ en abril de 1917, "día a día va ascendiendo el número de personas que se entregan a este renglón de explotación en nuestra ciudad", los que en la mayoría de los casos, afirmaba, eran ancianos y niños. ${ }^{11}$ La llegada del invierno no hacía más que agravar el problema, ya que "los primeros fríos [...] han hecho que la cantidad de mendigos, jóvenes y viejos, aumente en nuestra ciudad". ${ }^{12}$

9 "La mendicidad infantil en La Plata”, El Día, La Plata, 12 de julio de 1917. Algo que excedía a la provincia de Buenos Aires, como ha sido estudiado para Córdoba en un periodo similar, si bien con otros intereses ya que se busca reconstruir las imágenes sobre la mendicidad infantil, consultar Remedi (2019).

${ }^{10}$ Fundada en 1832, actualmente es cabecera del partido de Azul, y está ubicada a 300 kilómetros al suroeste de la Ciudad Autónoma de Buenos Aires. Es el centro geográfico de la provincia. De acuerdo con el censo de población de 1914, contaba para entonces con 32103 habitantes. Tandil, ubicada en el centro-oeste de la provincia, se encuentra a 350 de la capital, y para 1914 tenía 34061 habitantes según el Censo Nacional de Población.

11 "La mendicidad", El Ciudadano, Azul, 21 de abril de 1917.

12 "Aumento del mal", El Eco de Tandil, Tandil, 20 de mayo de 1917. 
Si bien no podemos sostener que denuncias de este tipo fueran una novedad del momento, se evidencia una especial insistencia en la gravedad que representaba el problema desde que, como El Ciudadano decía días después, "Se nota de un tiempo a esta parte, la reaparición de la tan debatida mendicidad infantil". ${ }^{13}$ También El Eco convenía en sostener que se trataba de un mal que "recrudece con caracteres más graves que en años anteriores" ${ }^{14} \mathrm{El}$ Día coincidía en esta tesitura y decía que "la plaga de la mendicidad infantil adquiere cada día que pasa, más graves caracteres". ${ }^{15}$

Cierto es que, como la misma prensa de distintas tendencias políticas ${ }^{16}$ coincidía en señalar, la situación económica era crítica y la mendicidad se agravaba. Afirmaba que "la actual guerra ha sido para el mendigo un acontecimiento fausto, pues ella ha venido a consolidar aún más la ya sólida y segura base en que se funda su condición social [...] desde un tiempo a esta parte ha tomado en nuestro país una progresión alarmante" máxime teniendo en cuenta, como sostiene tiempo después, "la situación económica tan calamitosa que se ha enseñoreado en todo el país". ${ }^{17}$

13 "Mendicidad infantil", El Ciudadano, Azul, 28 de mayo de 1917.

14 "Aumento del mal", El Eco de Tandil, Tandil, 20 de mayo de 1917.

15 "La mendicidad infantil", El Día, La Plata, 24 de enero de 1917. Sobre el incremento véase también "Vagancia infantil", La Nación, Buenos Aires, 21 de octubre de 1918. Sobre la vinculación entre calle, mendicidad y delincuencia, véanse "Niñez desamparada", La Nación, Buenos Aires, 23 de diciembre de 1917 y "Vagancia y mendicidad”, La Nación, Buenos Aires, 24 de septiembre de 1917.

${ }^{16}$ Aunque no podemos ignorar que la prensa y su denuncia de la indiferencia de los gobiernos municipales o provinciales no es ingenua ya que es un actor político que tomó partido, el tenor de los reclamos era similar en medios de prensa de orientaciones diversas como los analizados y los excedía.

El Eco de Tandil no se alineaba con ninguna facción política en particular, aunque defendía, cuando lo creía necesario, los intereses provinciales para lo que sostenía que había que dejar de lado los partidismos y las discrepancias particulares. El Ciudadano de Azul era radical y El Día de La Plata en este periodo tenía una clara tendencia contraria al gobernador Crotto. Sin embargo, debemos también tener en cuenta que la prensa estaba atravesada por las disidencias y facciones de los propios partidos. Así, El Ciudadano entre 1917 y 1922 era radical pero al mismo tiempo se oponía a la conducción del partido radical de Azul (Fuentes, 2016). El Día en 1913 apoyó por medio de su director Atencio, la designación de Ugarte como presidente del partido conservador, pero luego abandonó las filas para crear el Partido Provincialista. Al frente del mismo y a través del diario, denunció la existencia de la llamada "maquinaria ugartista" puesta al servicio del fraude. El Argentino, por su parte, fue crítico en relación al tema del Patronato durante el gobierno de Crotto y lo que consideraba la inacción oficial.

${ }_{17}$ "Un peligro", El Eco de Tandil, Tandil, 15 de abril de 1917 y "La hora actual", El Eco de Tandil, Tandil, 16 de septiembre de 1917. 
En efecto, esta coyuntura económica particular desatada a raíz de los cambios que provocó la guerra mundial en el equilibrio de los mercados internacionales, se insertó en una provincia de Buenos Aires que atravesaba una delicada situación económica y financiera, que diversos gobiernos habían enfrentado pero sin ser exitosos en su resolución definitiva (De Paz Trueba, 2018b).

Por ello, aunque no podemos aseverar que la evaluación que la prensa hacía sobre el aumento de la mendicidad respondiera a una situación novedosa, se insertaba en un contexto diferente que no hacía más que agravar viejas dificultades.

En este marco, se bregaba por la generación de nuevas formas de asistencia, que apuntaran no sólo a alimentar o vestir, sino también a regenerar por medio del trabajo. ${ }^{18}$ Desde fines del siglo xIx, legisladores, especialistas y miembros de distintas agencias gubernamentales propusieron diversas maneras de intervenir sobre sectores de la infancia que por su inserción familiar y social tenían un futuro poco promisorio. En relación con esas "conductas morales de la familia de pertenencia" y sus condiciones materiales, así como las trayectorias escolares y laborales de los niños, es que, como señala Stagno (2010), se propusieron nuevas "formas de reeducación y tratamiento" (p. 28). Entre otros aspectos, se vio en el trabajo una herramienta de moralización en algunos casos, y como regenerador en otros. Como ha sido señalado por diversos autores, el trabajo lejos de ser en este caso pensado como algo nocivo para la formación de los niños, como eran las ocupaciones callejeras, era una solución cada vez más pregonada porque se entendía que moldeaba la moral y la disciplina, además de que permitía adquirir conocimientos para el futuro. Un discurso que se acentuó en el periodo analizado en este trabajo (Aversa, 2015; De Paz Trueba, 2014 y 2017; Stagno, 2008; Zapiola, 2009). ${ }^{19}$

Los reclamos de la prensa estaban en sintonía con una matriz de pensamiento que atravesaba también a los actores políticos que debían gestionar las medidas. Tal era así que en su mensaje de 1916, el vicegobernador Vicente

18 "Los niños abandonados. Problemas sin solución seria”, El Día, La Plata, 1 de marzo de 1917. En esa misma línea coincidía La Nación. Véase "Vagancia infantil. Proyecto para cortarla", La Nación, 20 de marzo de 1917.

${ }^{19}$ No se trató de una situación particular de nuestro espacio. Como ha demostrado Susana Sosensky para el caso de México, la terapéutica del trabajo, fue parte de una idea propia del siglo XIX, que propugnaba el trabajo como forma de regeneración de delincuentes infantiles que obedeció "a la creación del nuevo tipo de ciudadano que requería el país: productivo, industrializado, trabajador y disciplinado" (Sosensky, 2008, p. 96). 
Peralta Alvear, ${ }^{20}$ afirmó que la Defensoría de Menores de la provincia no podía más que realizar "una labor superficial [...] el órgano no responde a la función y ésta necesariamente tiene que resultar débil, incompleta y anacrónica”. Por ello, sostuvo que era urgente trabajar en una nueva reglamentación de la misma, que desarrollaría su labor en forma mancomunada con el Patronato Provincial de Menores, que a todas luces era pensado como una manera novedosa y vanguardista de dar una solución definitiva al problema de la infancia y la minoridad abandonadas. ${ }^{21}$ En él, no sólo se asilaría sino también se formaría en oficios. La disponibilidad de espacios para instalar talleres y tierra para la práctica de la agricultura era uno de los ejes sobre los que el proyecto se asentaba. En suma, la Ley de Patronato provincial tenía por objeto resolver el gran problema que desde la óptica del gobernador y los legisladores, en coincidencia con la de la opinión pública, tenía la minoridad abandonada en la provincia. En los fundamentos de la misma, se sostenía que esos chicos y chicas no tenían más amparo en general que la "dirección pasiva y a menudo indiferente" de los Defensores de Menores locales. Por ello, y ante las dificultades de estos funcionarios del ámbito municipal de proteger efectivamente a esos pequeños que podían en un futuro cercano cometer delitos y transformarse en "parásitos sociales", la ley preveía crear una institución "destinada a albergar, educar y preparar para el trabajo y para una vida de orden y moralidad al mayor número posible de aquellos que se hallan abandonados en la provincia". Los destinatarios de la medida tenían entre siete y catorce años y podían permanecer en el asilo hasta los $18 .{ }^{22}$ Además de la insistencia en la formación de oficios, la modernidad de la medida a criterio de sus promotores residía en que por primera vez el Estado tomaba en sus manos la función de atender de manera directa y con mayor alcance a la infancia abandonada, dado que hasta entonces eran sólo las instituciones privadas las que se hacían cargo de medidas semejantes, aunque de manera insuficiente..$^{23}$

${ }^{20}$ En ejercicio del poder porque el gobernador Marcelino Ugarte había delegado en él la administración de la provincia.

${ }^{21}$ Diario de Sesiones de la Provincia de Buenos Aires. Año 1916. Cámara de Senadores. Sesión del 8 de mayo de 1916. La Plata, Taller de impresiones oficiales, 1916, pp. 58-59. Archivo de la Legislatura de la provincia de Buenos Aires (en adelante ALPBA), La Plata, Argentina.

22 Diario de Sesiones de la Provincia de Buenos Aires. Año 1910. Cámara de Senadores. Sesión del 4 de octubre de 1910. La Plata, Taller de impresiones oficiales, 1910, pp. 559-562. ALPBA, La Plata, Argentina.

${ }^{23}$ Diario de Sesiones de la Provincia de Buenos Aires. Año 1910. Cámara de Senadores. Sesión de prórroga del 17 de octubre de 1910. La Plata, Taller de impresiones oficiales, 1910, pp. 575-582. ALPBA, La Plata, Argentina. 


\section{EL PATRONATO EN TIEMPOS DEL RADICALISMO. DE AVANCES Y DEMORAS}

Ahora bien, ¿qué sucedía por entonces con esta vieja aspiración que era reclamada como urgente pero seguía sin llevarse a cabo?

Algunos progresos se dieron en épocas del segundo gobierno de Ugarte..$^{24}$ Fue durante esta gestión que se propuso instalar el Patronato en una fracción de tierra fiscal ubicada en la sección Abasto de la ciudad de La Plata, algo que suponía un avance de consideración puesto que "uno de los obstáculos con que ha tropezado la realización de la idea ha sido precisamente la adquisición de la tierra". ${ }^{25}$

Al llegar el interventor nacional al poder de la provincia tomó decisiones que fueron luego retomadas por el gobernador que le sucedió. En ese marco reglamentó el funcionamiento de lo que se llamó depósito del patronato, un local que había sido reservado por el gobierno de Ugarte para refugio de niños ubicado en la calle 6 número 1274 de la ciudad, que debía funcionar de manera provisoria hasta tanto se instalara el instituto definitivo. ${ }^{26}$

En 1918, el interregno de Cantilo dio paso a la llegada de Crotto al poder de la provincia, luego de una ardua negociación entre las dos facciones internas del partido para designar candidatos. La fórmula finalmente elegida que involucraba a un miembro de cada una de ellas pareció inaugurar una época de tranquilidad política e institucional. ${ }^{27}$ Sin embargo, apenas llegado

${ }^{24}$ Marcelino Ugarte ocupó por segunda vez la gobernación provincial entre mayo de 1914 y abril de 1917, cuando fue reemplazado por el interventor designado por el presidente Yrigoyen.

${ }^{25}$ Registro Oficial de la Provincia de Buenos Aires. Año 1916. Tomo 1, enero a junio. La Plata, Taller de Impresiones Oficiales, 1916. Departamento de gobierno. Decreto núm. 273. 15 de mayo de 1916, pp. 264-266. ALPBA, La Plata, Argentina.

${ }^{26}$ Registro Oficial de la Provincia de Buenos Aires. Año 1917. Tomo 2, julio a diciembre. La Plata, Taller de Impresiones Oficiales, 1919. Intervención nacional. Departamento de gobierno. Decreto núm. 619, 4 de septiembre de 1917, pp. 634-640. ALPBA, La Plata, Argentina.

${ }^{27}$ Las dificultades para conformar la fórmula radicaron principalmente en las divisiones internas de la Unión Cívica Radical, particularmente entre dos facciones: provincialistas y metropolitanos. Esta rivalidad, que no expresaba diferencias doctrinarias sino que representaba dos maneras de entender la relación entre el gobernador de la provincia de Buenos Aires con el presidente de la nación, no era nueva en la provincia. Los avances de los políticos nacionales sobre los destinos de la provincia habían sido siempre resistidos por los políticos bonaerenses. Los primeros defendían los intereses de la provincia y los metropolitanos tenían una alianza más definida con los lineamientos del poder nacional. Estas diferencias se hicieron manifiestas en las filas del radicalismo tras la llegada al poder de Yrigoyen, que hasta entonces y en tanto 
al poder, Crotto comenzó a tomar decisiones de manera autónoma respecto del presidente Yrigoyen y las rispideces surgidas entre ambos tornaron irreconciliable la fractura dentro de la Unión Cívica Radical (UCR). ${ }^{28}$

Entre tanto, la cuestión de los niños mendigos y abandonados no había sido resuelta con la instalación del refugio. Como recordaba El Día, "no debe olvidarse, que lo que funciona en la calle 6 entre 58 y 59, es un depósito provisorio del Patronato, inaugurado en octubre de 1917 con 63 asilados sobre los 600 o más que según la memoria del interventor nacional, tienen a su cargo los defensores generales de menores, número muy inferior a los que carecen de toda protección en el extenso territorio de la provincia". ${ }^{29}$

Meses antes, El Argentino se había manifestado en el mismo tenor al señalar que esa solución provisoria que fue completada por la Intervención, no se desarrollaba de la mejor manera, a juzgar por los hechos que se sucedían en su vida cotidiana. Decía que "la normalidad no es la característica en el desenvolvimiento del refugio. Tumultos, fugas, reclamaciones, se destacan en la historia brevísima de la institución. Sus asilados no se sienten cómodos,

oposición al régimen conservador habían marchado unidos bajo su conducción. Elegido presidente, se convirtió en metropolitano al querer que los destinos de la provincia estuvieran bajo su influencia a través de hombres de su estricta confianza. La fórmula Crotto-Monteverde expresó un compromiso ante las filas radicales en tanto Crotto representaba a los metropolitanos y Monteverde a los provincialistas. No obstante, como ha sostenido Barba, fue inevitable que algunos de aquellos que habían acompañado a Yrigoyen una vez llegado a la presidencia, no aceptaran el intento del caudillo de mantener su liderazgo. Como veremos fue el caso de Crotto quien mantendría una posición propia tras llegar al gobierno de Buenos Aires (Barba, 2007).

${ }^{28}$ Como afirma Fernando Barba, la causa de la ruptura fueron las designaciones de funcionarios afines a Crotto, sacando en muchos casos los que en diversas reparticiones oficiales y en número considerable había nombrado el mismo interventor Cantilo durante los dos últimos meses de su gestión. El problema, a decir del autor citado, era en suma de "quien designaba a los funcionarios, y a la larga de quien mandaba, y no por motivos ideológicos o por luchas de sectores políticos o económicos" (Barba, 2010, p. 54). Las cesantías ordenadas por Crotto, sumadas a otros nombramientos y su intención de actuar en forma autónoma habría generado malestar tanto entre los partidarios de Yrigoyen que querían mantener su injerencia sobre todos los gobernadores, como entre los mismos provincialistas (partidarios de Crotto en un primer momento), dejando con el curso de los meses al gobernador en soledad y al partido dividido aun al interior de sus mismas facciones. Una práctica que según ha sostenido Salvatore (2016), se habría visto aumentada durante los gobiernos radicales. El excesivo uso del empleo público como herramienta para expandir la clientela política habría además atentado contra la conformación de burocracias especializadas a partir de la meritocracia.

29 "El patronato de menores", El Día, La Plata, 23 de octubre de 1918. 
satisfechos, atraídos por el calor de la casa común. Lejos de eso, reciben la sensación del presidio, se quejan del tratamiento que reciben." 30

Funcionaba en una casa precaria que daba cama y alimento a un número reducido de menores, algo que "puede decirse que nada tiene que ver con la institución del Patronato que está en el espíritu de la ley [...] que sugiere una idea más alta que la del refugio o el asilo". ${ }^{31}$

Si bien como se señaló antes durante la gobernación de Ugarte y Peralta Alvear se había destinado un terreno para la construcción del edificio del Patronato que debía albergar a niños de toda la provincia y propendiera su formación en oficios, en 1918 eso aún no se había concretado. Más aún, según relataba El Día, gracias a la oportuna intervención del diputado radical Vicente Damonte, se pudo evitar que el terreno fuera puesto en remate. ${ }^{32}$ En julio de 1918, Damonte presentó un proyecto a la Cámara para impedirlo y además declaró que era un "bien público e inajenable con destino al Patronato Provincial de Menores de ambos sexos la fracción de tierra fiscal ubicada en la sección Abasto del ejido de la ciudad". Al mismo tiempo el proyecto contemplaba que el poder ejecutivo diera "comienzo de inmediato a la construcción del edificio [...] con los fondos destinados a dicho fin". ${ }^{33}$ Finalmente, y dado que las instalaciones del refugio de la calle 6 no estaban en buenas condiciones, proponía trasladar a los chicos allí alojados a otro lugar mientras se erigía el Patronato. En sintonía con esta idea, ese mismo mes el gobernador Crotto firmó un decreto por el cual dispuso el traslado de esos menores a la llamada Cárcel Mecánica, por disponer allí de mayor espacio. Al mismo tiempo, el anterior depósito del Patronato sería destinado al alojamiento de las menores de sexo femenino que aún permanecían en la cárcel de mujeres y otros establecimientos, a pesar de que el proyecto original del Patronato concebía que este debía ser para ambos sexos. ${ }^{34}$ Con la medida, se trasladarían los chicos del refugio y "Desocupado el local del Patronato de la calle 6, este sería ocupado

30 "El Refugio de Menores", El Argentino, La Plata, 25 de marzo de 1918.

31 "Patronato de Menores", El Argentino, La Plata, 25 de marzo de 1918.

32 "Patronato platense de menores. El proyecto del diputado Damonte", El Día, La Plata, 14 de julio de 1918.

${ }_{33}$ Diario de Sesiones de la Provincia de Buenos Aires. Año 1918. Cámara de Diputados. Sesión del 17 de julio de 1918. La Plata, Taller de impresiones oficiales, 1919, pp. 637-638. ALPBA, La Plata, Argentina.

${ }^{34}$ Registro Oficial de la Provincia de Buenos Aires. Año 1918. Tomo 1, enero a junio. La Plata, Taller de Impresiones Oficiales, 1919. Departamento de gobierno. Decreto núm. 313, 11 de julio de 1918, p. 819. ALPBA, La Plata, Argentina. 
por las menores recluidas en la cárcel de mujeres que viven en promiscuidad con las penadas." Esta medida permitiría "mejorar la situación penosa de los menores depositados por la policía y en la cárcel de mujeres a la vez que ganarán del Patronato, hacinados en local insuficiente por su número". ${ }^{35}$

Damonte explicaba en sus fundamentos que si en ocasión de ser sancionada la ley de creación del Patronato en 1910, esta era necesaria, "Hoy es indispensable, pues la provincia de Buenos Aires solo cuenta para alojamiento de los menores abandonados, la pequeña casa de esta ciudad [que] tiene apenas capacidad para sesenta niños". Teniendo en cuenta -según señalabaque la ley disponía de los medios para destinar recursos para la construcción (ya que estos habían sido descontados a las municipalidades), existían fondos para tal fin, aunque no se había tomado la decisión política de hacerlo. ${ }^{36}$ Por todo ello, decía: "ha llegado, entonces, el momento de hacer efectiva la construcción del primer gran instituto modelo que ha de resolver el problema de dar albergue, alimentación, vestido y educación a todos los niños huérfanos y abandonados en la provincia de Buenos Aires." ${ }^{\text {37 }}$

El mensaje de Damonte era optimista puesto que creía que esta institución vendría a terminar con la delincuencia infantil en buena parte, cuando "los defensores de menores no tengan que mendigar y repartir entre las

35 "Los menores recluidos", El Argentino, La Plata, 6 de junio de 1918.

${ }^{36}$ Tanto El Argentino como El Día y los legisladores que se ocuparon del tema en las Cámaras, coincidían en señalar que no se trataba de un problema económico sino de una dilación política. La ley que creó el Patronato había también previsto la manera de sostenerlo, con aportes de las municipalidades que luego podrían enviar allí chicos y chicas. Esos descuentos, tal como la prensa y los legisladores denunciaban, habían sido hechos desde 1912, razón por la cual existía un fondo con el que creían que se podía afrontar la obra. También el Registro Oficial permite corroborar la manera en que se planificaba la utilización de estos fondos en los presupuestos de años sucesivos. Esto permite sugerir que si bien la provincia no atravesaba por un buen momento financiero, es posible que se contara efectivamente con el dinero, y es por ello que se deben buscar razones más complejas que expliquen la demora en su instalación. "Patronato platense de menores. El proyecto del diputado Damonte", El Día, La Plata, 14 de julio de 1918; "Patronato de Menores", El Argentino, La Plata, 25 de marzo de 1918, entre otros. Diario de Sesiones de la Provincia de Buenos Aires. Año 1918. Cámara de Diputados. Tomo I. Sesión del 11 de julio de 1918. La Plata, Taller de Impresiones Oficiales, 1919, pp. 637-638; Diario de Sesiones de la Provincia de Buenos Aires. Año 1918. Cámara de Senadores. Sesión del 8 de octubre de 1918. La Plata, Taller de Impresiones Oficiales, 1919, pp. 1034-1036. ALPBA, La Plata, Argentina.

${ }^{37}$ Diario de Sesiones de la Provincia de Buenos Aires. Año 1918. Cámara de Diputados. Sesión del 11 de julio de 1918. La Plata, Taller de Impresiones Oficiales, 1919, pp. 637-638. ALPBA, La Plata, Argentina. 
familias a los menores desamparados que abundan en todas las comunas". Reconocía que "habremos dado un gran paso". ${ }^{38}$

A pesar de la importancia del proyecto, que fue aprobado, la construcción no se inició y tampoco fueron instaladas las menores en la calle 6. La Cámara de Senadores sería escenario de otra batalla más ese mismo año. En octubre de 1918, el senador radical José Cabral presentó una minuta de comunicación pidiendo una interpelación al poder ejecutivo por las obras del Patronato. ${ }^{39}$ Fundamentaba esta petición diciendo que "Dada la trascendencia del asunto [...] es absolutamente necesario llamar la atención de los poderes públicos y del pueblo mismo sobre uno de los aspectos de nuestra vida que, en verdad, nos presenta como una sociedad inorgánica, como un país sumamente pobre [...]. No obstante esa ley de 1910, nada en realidad se ha hecho hasta el año pasado." ${ }^{\prime 0}$

Con esto hacía referencia a las acciones de Peralta Alvear y del interventor Cantilo al instalar y reglamentar el refugio de la calle 6. Ese lugar seguía, según afirmaba, en las mismas condiciones que tras su creación "donde los menores se hallan poco menos que apilados [...] apenas es posible enseñarles a leer y escribir [...] no hay talleres, no se les puede enseñar ningún oficio". ${ }^{4}$ Mientras, sostenía que había más de 600 niños en poder de los defensores en toda la provincia que no sabían dónde colocarlos. Para Cabral, en consonancia con un clima de ideas generalizado entre las elites políticas e intelectuales de la época, este era un problema urgente que debía resolverse. Como decía El Argentino, "Poseyendo los medios requeridos, no tiene explicación satisfactoria el retardo observado en la realización de la idea. No se dirá que hayan disminuido o que falten las necesidades que lo imponen. Por el contrario las razones [...] perduran [...]. Se clama frecuentemente contra el aumento de la

${ }^{38}$ Diario de Sesiones de la Provincia de Buenos Aires. Año 1918. Cámara de Diputados. Sesión del 11 de julio de 1918. La Plata, Taller de Impresiones Oficiales, 1919, pp. 637-638. ALPBA, La Plata, Argentina.

${ }^{39}$ En el marco de las rivalidades entre Crotto e Yrigoyen, la interpelación fue una herramienta parlamentaria usada para obstaculizar al gobierno de Crotto por opositores tanto fuera como dentro del radicalismo. Según Barba, esto muestra además, la pervivencia de viejas prácticas políticas tan denostadas por los propios radicales (Barba, 2009).

${ }^{40}$ Diario de Sesiones de la Provincia de Buenos Aires. Año 1918. Cámara de Senadores. Sesión del 8 de octubre de 1918. La Plata, Taller de Impresiones Oficiales, 1919, pp. 1034-1036. ALPBA, La Plata, Argentina.

${ }^{41}$ Diario de Sesiones de la Provincia de Buenos Aires. Año 1918. Cámara de Senadores. Sesión del 8 de octubre de 1918. La Plata, Taller de Impresiones Oficiales, 1919, pp. 1034-1036. ALPBA, La Plata, Argentina. 
mendicidad y de la vagancia, a los que aporta un apreciable contingente el elemento infantil." 42

A medida que pasaban los meses, la oposición al gobierno de Crotto se hacía más intensa y las quejas sobre esta y otras cuestiones arreciaban, tanto desde la opinión pública en general como desde el radicalismo. Una vez más, se acusaba al gobierno (como antes a otros) de negligencia y se subrayaba la responsabilidad política que le cabía al respecto: "el poder ejecutivo siguiendo la conducta de los anteriores gobiernos, no se preocupa, al parecer en lo más mínimo de solucionar aquella importante cuestión [...] la solución referida no se busca sencillamente por desidia, pues como se sabe existen todos los medios necesarios para hallarla" ${ }^{43}$

Aludía con esto a la ley sancionada, los recursos que año con año se habían ido reteniendo a los municipios, los planos aprobados y el terreno designado esperando ver levantarse allí las paredes del instituto. Por todo ello, era enfático al afirmar que "el gobierno del Dr. Crotto tiene el deber de iniciar las obras del proyectado patronato". ${ }^{44}$ Si bien como se adelantó páginas antes, siguiendo a Fernando Barba (2009), la interpelación parlamentaria fue una herramienta usada con fines políticos opositores y habría servido para desgastar al gobierno provincial y no para discutir temas centrales que se vieron así postergados. Las fuentes analizadas permiten sugerir que el Patronato ocupó un lugar en ellas, reflejando una preocupación constante que iba más allá de estas intenciones partidarias.

\section{HACIA 1920: INICIATIVAS, MEDIDAS Y CONCRECIONES}

Como se afirmó, estas cavilaciones no sólo eran producto de las páginas de la prensa opositora. La preocupación por el Patronato, en particular, y por otras acciones en pro de resolver el tema de la infancia abandonada, pobre y mendicante era transversal a los diversos medios de prensa aquí estudiados y a profesionales de distintas disciplinas (Stagno, 2010; Zapiola, 2014). Aquella sin

\footnotetext{
42 "Patronato de Menores", El Argentino, La Plata, 22 de noviembre de 1918.

43 "De la obra del gobierno. Instalación del Patronato de Menores", El Día, La Plata, 6 de mayo de 1919.

44 "De la obra del gobierno. Instalación del Patronato de Menores", El Día, La Plata, 6 de mayo de 1919.
} 
duda contribuyó a generar una mayor presión sobre el tema, pero el mismo estaba instalado también en el recinto de ambas cámaras. Antes señalamos que había sido el diputado Damonte quien gracias a su "oportuna actitud" había evitado el remate de las tierras, situación generada a causa del "desconocimiento que en las altas esferas oficiales parecería haber imperado". ${ }^{45}$

También había sido un radical, el senador Cabral, quien había usado la herramienta de la interpelación política para poner el tema sobre la mesa. ${ }^{46}$ Pero ante la demora, en 1919 un grupo de diputados conservadores y socialistas (Arana, Amoedo, Lemos y Baliño) fueron los que tomaron el asunto en sus manos. Como primera medida visitaron el refugio, y según relataba El Día "oímos de sus labios las más dolorosas impresiones recibidas y los calificativos más duros en cuanto a la situación a que se había llegado en aquella casa”. Entre los detalles que el diario ofrece, se habla de falta de higiene, de buena alimentación, hasta de la presencia de sarna en algunos chicos, dolencias que no eran tratadas de la manera adecuada. ${ }^{47}$ Por ello, decidieron elevar otro pedido de interpelación por el mismo tema. Así, exigieron que el ministro de Gobierno informara acerca de

qué medidas ha tomado el poder ejecutivo para subsanar las deficiencias de organización y funcionamiento del depósito del Patronato de Menores que funciona en la calle $6[. .$.$] ante las denuncias hechas por la prensa diaria y$ que son del dominio público [...] cuál es el estado actual del establecimiento y qué piensa el Poder Ejecutivo con respecto a la instalación definitiva del Patronato de Menores. ${ }^{48}$

45 "El Patronato de Menores", El Día, La Plata, 23 de junio de 1919.

${ }^{46}$ Cabe señalar que Cabral se desempeñó con posterioridad a esta intervención, como ministro de gobierno de Crotto, pero no por su cercanía con el gobernador sino como parte de una estrategia para lograr una conciliación que permitiera la gobernabilidad. En enero de 1921, Crotto nombró tres ministros, entre ellos Cabral, sugeridos por la cúpula partidaria. La crisis pareció resolverse pero sólo de manera momentánea ya que en marzo comenzaron a llegar críticas de los ministros que decían que el gobernador ni siquiera se reunía con ellos. En abril fueron destituidos por Crotto, quien en mayo presentó su renuncia (Fuentes, 2016). Como sostiene Barba, ese acercamiento fue uno de muchos que se "hicieron sobre bases imposibles de producir efectos positivos ya que ninguna de las partes estaba dispuesta, pese a las declaraciones en sentido contrario a ceder en sus pretensiones" (Barba, 2010, pp. 77).

47 "El refugio de menores. Una vergüenza para La Plata", El Día, La Plata, 21 de agosto de 1919; y “El Refugio de menores”, El Día, La Plata, 22 de agosto de 1919.

${ }_{48}$ Diario de Sesiones de la Provincia de Buenos Aires. Año 1919. Cámara de Diputados. Sesión del 20 de agosto de 1919. La Plata, Taller de Impresiones Oficiales, 1919, p. 1247. ALPBA, 
No obstante las preocupaciones manifestadas, y las solicitudes de pronta resolución del asunto, cabe preguntarse qué medidas efectivas se tomaron en el gobierno de Crotto en favor del Patronato. ¿Existió realmente tal desidia política, tal desinterés como se denunciaba?, ¿debemos ver en esas denuncias un interés político en denostar antes que una verdadera preocupación por los menores desamparados?, ¿eran estos una inquietud para Crotto o tenía en su agenda otras prioridades?

Como se señaló más atrás, las críticas provenían de distintos medios escritos (opositores y otros independientes) y políticos de diferentes partidos, quienes reflejaban un malestar por la denominada infancia desamparada que no era nuevo y que la prensa y diversos sectores intelectuales y profesionales venían manifestando hacía años. Tal es así que los estudios de historia de la infancia han mostrado de qué manera la Ley de Patronato de Menores de 1919 habría sido un punto de llegada, al dar estatuto legal a intervenciones sobre la infancia pobre que habían sido reclamadas al menos desde 1890 y que se habían reflejado en artículos periodísticos, proyectos legislativos y comunicaciones en eventos científicos. Todo lo cual trascendía el ámbito nacional (Zapiola, 2014; Stagno, 2010).

Sin embargo, el edificio definitivo no se erigió durante este primer gobierno radical y fue a mediados de 1920, meses antes de renunciar al cargo, cuando el gobernador Crotto en medio de grandes presiones de la prensa y del medio político puso la piedra fundacional del Patronato. ${ }^{49}$

¿Tuvo que ver esta demora con las prioridades del gobierno de Crotto?, ¿cuáles eran estas? Tanto en sus últimas actuaciones en el Senado nacional donde se desempeñó (antes de ser gobernador) en representación de la Ca-

La Plata, Argentina. Esta interpelación fue contestada punto por punto por el gobernador, y se dio lectura a la misma en la sesión del 3 de septiembre. Allí decía Crotto que "El Poder Ejecutivo desde sus primeros días de gobierno se dio cuenta exacta de las deficiencias que adolecía el Patronato", y respondía por ello detallando todo lo que había hecho al respecto. Señalaba entre otras cosas, las gestiones hechas para trasladar a los menores a otro local más amplio, así como a la selección de otro personal a los fines de mejorar el funcionamiento interno. Además, decía que "No se desoyeron las denuncias de la prensa diaria. Antes bien, se reconoció la buena intención de sus justas críticas". Diario de Sesiones de la Provincia de Buenos Aires. Año 1919. Cámara de Diputados. Sesión del 3 de septiembre de 1919. La Plata, Taller de Impresiones Oficiales, 1919, pp. 1445-1446. ALPBA, La Plata, Argentina.

49 "Patronato de Menores. Cumplimiento de la ley de 1910", El Día, La Plata, 13 de mayo de 1920; "Patronato de menores. La construcción del edificio", El Día, La Plata, 21 de mayo de 1920; "Patronato de menores. Primera piedra del futuro edificio", El Día, La Plata, 21 de junio de 1920. 
pital Federal, como en su gira política consagrado ya como candidato a la primera magistratura de la provincia, no parece ser la infancia pobre un problema que ocupara su interés. ${ }^{50}$ Es posible en cambio afirmar que la justicia, la administración de la provincia en particular en lo relativo a su régimen municipal, los ferrocarriles, la instrucción pública, la vialidad, la ganadería y la agricultura, fueron los puntos en los que hizo hincapié y en los que se comprometió a trabajar si lograba ser electo gobernador.

Crotto era consciente de que llegaría a la gobernación en un momento delicado en cuanto al panorama internacional, así como al estado en que las finanzas de la provincia se encontraban. Decía en su discurso en San Nicolás que "Nos encontramos con una situación financiera enferma, producto de la imprevisión, del derroche y del desbarajuste reinante en la provincia de Buenos Aires." ${ }^{11}$ Por lo tanto, proponía hacer un nuevo padrón de contribuyentes y establecer un nuevo cálculo de la valuación de la propiedad. Pero la clave de la recuperación entendía que estaba en el campo y su potencial productivo: "necesitamos hacer al pueblo rico [...] y esto solamente lo conseguiremos desarrollando con amplitud, nuestras grandes fuentes de producción", ${ }^{52}$ para lo cual entendía que era necesario dar impulso no sólo al desarrollo crediticio que permitiera producir, sino también, y sobre todo, remediar la carencia de infraestructura, especialmente caminos. Sostenía sin dudar la importancia de construir "grandes y sólidas carreteras [ya que] es una necesidad reclamada imperiosamente por nuestra producción y nuestra prosperidad". ${ }^{53}$

${ }^{50}$ Refiero especialmente a discursos y proyectos presentados en 1917-1918. Véase José Camilo Crotto. Discursos pronunciados por el Senador Nacional y candidato a gobernador Dr. José Camilo Crotto en el Senado de la Nación y en su gira política por la Provincia de Buenos Aires. Publicación del Comité Nacional de la Unidad Cívica Radical, 1917-1918. Archivo del Comité Nacional de la UCR (en adelante ACN de la UCR), Buenos Aires, Argentina.

${ }^{51}$ José Camilo Crotto. Discursos pronunciados por el Senador Nacional y candidato a gobernador Dr. José Camilo Crotto en el Senado de la Nación y en su gira política por la Provincia de Buenos Aires. Publicación del Comité Nacional de la UCR, 1917-1918, p. 140. ACN de la UCR, Buenos Aires, Argentina.

${ }_{52}$ Discurso pronunciado en la ciudad de La Plata la noche de la proclamación de la candidatura de Crotto para gobernador, p. 112. En José Camilo Crotto. Discursos pronunciados por el Senador Nacional y candidato a gobernador Dr. José Camilo Crotto en el Senado de la Nación y en su gira política por la Provincia de Buenos Aires. Publicación del Comité Nacional de la UCR, 19171918. ACN de la UCR, Buenos Aires, Argentina.

${ }_{53}$ Discurso pronunciado en Bahía Blanca, p. 120. En José Camilo Crotto. Discursos pronunciados por el Senador Nacional y candidato a gobernador Dr. José Camilo Crotto en el Senado de la Nación y en su gira política por la Provincia de Buenos Aires. Publicación del Comité Nacional de la UCR, 1917-1918. ACN de la UCR, Buenos Aires, Argentina. 
En estrecha relación con lo anterior, le preocupaba el tema de las inundaciones y por ello proponía trabajar en obras públicas que permitieran el desagüe en épocas de lluvias, así como aprovechar aquellas aguas que pudieran reservarse en "represas artificiales en las épocas de abundancia, a fin de devolver el precioso elemento en la época amarga de la sequía". ${ }^{54}$

En todas estas alocuciones, hubo pocas referencias a los menores, y especialmente a aquellos que debía socorrer el Patronato. Si bien concordaba con el sentir general de la necesidad de brindar una formación práctica que preparara a los niños en trabajos acordes con las necesidades productivas de la nación, esto no tenía que ver con la formación de aquellos que no contaban con familia, ni recursos, sino que debe interpretarse dentro de su interés más general: sacar a la provincia del estado de decadencia económica en que se encontraba por entonces, apostando a ello con la producción del campo, lo que entendía era el mejor recurso disponible. De allí que en su programa está contenida también una reforma a la educación elemental. En relación con esto, pensaba que "no debe demorarse la difusión de los conocimientos prácticos por medio de escuelas y cursos análogos a los que constituyen la instrucción técnica en el viejo mundo y en Estados Unidos". ${ }^{55}$ Finalmente, no pensaba en el asistencialismo como algunos de sus congéneres lo estaban haciendo entonces, sino que propugnaba la instalación de colonias para niños débiles, donde la intervención de los médicos haría un bien por el "cuidado de la infancia desvalida". ${ }^{56}$

Vale a esta altura preguntarse qué fue lo que pudo llevar a cabo de las ideas con las que llegó al poder. Si bien estuvo al frente de un gobierno de rumbo azaroso, en razón de las vicisitudes políticas a las que se vio expuesto, no por ello se mantuvo inactivo. Tal como Barba ha sostenido, pese a las nu-

${ }^{54}$ Discurso pronunciado en Bahía Blanca, pp. 124-125. En José Camilo Crotto. Discursos pronunciados por el Senador Nacional y candidato a gobernador Dr. José Camilo Crotto en el Senado de la Nación y en su gira política por la Provincia de Buenos Aires. Publicación del Comité Nacional de la UCR, 1917-1918. ACN de la UCR, Buenos Aires, Argentina.

${ }_{55}$ Discurso pronunciado en Chivilcoy, p. 153. En José Camilo Crotto. Discursos pronunciados por el Senador Nacional y candidato a gobernador Dr. José Camilo Crotto en el Senado de la Nación y en su gira política por la Provincia de Buenos Aires. Publicación del Comité Nacional de la UCR, 1917-1918. ACN de la UCR, Buenos Aires, Argentina.

${ }^{56}$ Discurso pronunciado en Chivilcoy, p. 156. En José Camilo Crotto. Discursos pronunciados por el Senador Nacional y candidato a gobernador Dr. José Camilo Crotto en el Senado de la Nación y en su gira política por la Provincia de Buenos Aires. Publicación del Comité Nacional de la UCR, 1917-1918. ACN de la UCR, Buenos Aires, Argentina. 
merosas y permanentes trabas que encontró en el poder legislativo, su acción de gobierno no fue despreciable tanto en materia de pago de deuda externa, de obras viales, obras sanitarias, refacciones de hospitales, obras de desagües, entre otras (Barba, 2010). Según afirma Moreyra (2009) para el caso de Córdoba, los gobiernos no habrían estado tan dispuestos a invertir dinero y a gestionar medidas políticas para atender las cuestiones sociales como a otras relativas a consolidar las estructuras institucionales del Estado, fortalecer el aparato de seguridad y la expansión de la infraestructura física que garantizara el crecimiento económico. En el caso analizado vimos que los retrasos y vaivenes no pueden vincularse exclusivamente a la cuestión económica. No obstante esto, es cierto que las convicciones del gobernador (expuestas en su gira política) se condicen con esa tendencia que observa Moreyra, mas no con la preocupación por los niños que debía atender el Patronato, tan presente en otros medios.

Por lo demás, y ya en su cargo de gobernador, fue en 1920 en su discurso para la Legislatura cuando Crotto manifestó en la Cámara su preocupación por el Patronato. Decía que "Para amparar y tutelar a los menores que por diversas causas deben depender del Patronato, no basta sólo con la buena voluntad [...] Es necesario crear un instituto de preservación y de reforma [...] para hacer de esos infelices sin familia y sin hogar, organismos aptos para el trabajo fecundo y regenerador." Teniendo en cuenta, sostenía, que la delincuencia infantil se relacionaba más con el ambiente en el que los niños crecían y se desarrollaban que con causas atávicas, el Patronato era fundamental en la provincia de Buenos Aires. Así, "desde los primeros días de mi gobierno, comprendí que era necesario prestarle a esta Institución la mayor atención posible". El Patronato estaba instalado en "un local inadecuado y su personal requería una selección enérgica [...]. De una manera lenta pero constante el Poder Ejecutivo fue realizando esas reformas." ${ }^{57}$ Era consciente, sin embargo, de que esto no alcanzaba para hacer realidad todos los preceptos de la Ley que diera origen al proyecto en el año 1910. Creía que en breve podría el "Instituto

${ }^{57}$ Al respecto, es posible señalar que el Registro Oficial de la Provincia de Buenos Aires muestra entre 1918 y 1921 una enorme cantidad de cesantías y nombramientos de empleados de distintos rangos en el Patronato Provincial de Menores. Sin embargo, no podemos asegurar que estos se debieran a un interés genuino del gobernador por mejorar la selección de ese personal tal como él manifiesta, o que en cambio se tratara de un uso del empleo público como prebenda política, como ha sido estudiado por varios de los autores citados. Una tendencia que de acuerdo con Salvatore (2016) se habría visto acrecentada durante los gobiernos radicales. 
en su edificio propio [...] responder ampliamente al noble objetivo", lo que se vería cumplido cuando comenzara su instalación. ${ }^{58}$

Tal como señalamos, Crotto colocó la piedra fundamental poco después de este mensaje y llamó a licitación para la compra de 8000000 de ladrillos. No obstante, años después seguían estos sin ser usados para el fin con que habían sido adquiridos. ${ }^{59}$ Tal era así que El Día señalaba que pasados varios meses de colocada la piedra fundacional "en medio de pomposa ceremonia oficial en los terrenos designados, a inmediaciones del Abasto y a los diez años de promulgada la ley de creación de utilísimo y anhelado establecimiento. Nada se ha hecho, con carácter definitivo en favor de la erección que aquella ceremonia consagró." La fuente sostenía que se contaba con el dinero aunque el fondo disponible para la construcción al que aportaban las municipalidades había disminuido a causa de "los desgraciados ensayos de Patronatos provisorios". De todos modos, era suficiente para dar comienzo a la obra. Es así que "Falta solo un poco de actividad para que por fin veamos surgir el Patronato de Menores como se concibió y como quisieron que se formara el Poder Ejecutivo y el Poder Legislativo hace diez años, con el objeto que tuviera principio de ejecución la solución del problema de la niñez desamparada de la provincia." ${ }^{60}$

No obstante los dichos del diario platense, esos ensayos del Patronato constituyeron en la práctica la respuesta concreta a la situación de la infancia que, de acuerdo con la Ley de 1910, se había dado desde el poder ejecutivo. Aunque incompletos y tardíos tal como los mismos representantes del gobierno reconocían, permitieron poner manos a la obra, en primer término en el caso de los varones. Pero además, poco después también durante la gestión de Crotto en 1920, y como parte de las medidas tomadas durante su gobierno, se avanzó en el amparo de menores de sexo femenino. La Ley de 1910 contemplaba asilar y formar a menores de ambos sexos. Sin embargo, el antecedente del Patronato de Abasto, el Refugio de Menores que funcionó en la calle 6, fue en un primer momento, como se señaló antes, sólo para varones. Hacia 1918,

${ }^{58}$ Mensaje del gobernador de la provincia de Buenos Aires, doctor José Camilo Crotto, dirigido a la Asamblea Legislativa el 1 de mayo de 1920. La Plata, Taller de Impresiones Oficiales, 1920, pp. 18-19. AlPBA, La Plata, Argentina.

${ }_{59}$ "Patronato Provincial de Menores. ¿Cuándo se iniciarán las obras?”, El Día, La Plata, 27 de marzo de 1921.

${ }_{60}$ “Patronato Provincial de Menores. ¿Cuándo se iniciarán las obras?”, El Día, La Plata, 27 de marzo de 1921. 
por primera vez, se retomó de manera oficial la cuestión de las mujeres. Así, rezaban los considerandos de un decreto del gobernador Crotto que sostenía que "el local del Depósito del Patronato de menores [...] resulta insuficiente para alojar a los menores desamparados [...] que a fin de resolver el problema de la niñez desamparada es de impostergable necesidad habilitar los locales adecuados para alojar a los menores de ambos sexos". Por ello como se adelantó páginas antes, decretó el traslado de los varones al local de la llamada Cárcel Mecánica, para dejar libre el local de la calle 6 a las mujeres menores. ${ }^{61}$

En 1920 el proyecto no se había concretado, y poco antes de renunciar el gobernador Crotto, se insistió en él. Así, otro decreto provincial estableció el alojamiento de mujeres en la calle 6, encargando de la administración, educación, cuidado y vigilancia de las menores a la Congregación de Nuestra Señora de la Misericordia. ${ }^{62}$ Sin embargo, tras la renuncia de Crotto aún no se hacía efectivo el decreto, a pesar de que, como decía una resolución del Departamento de Gobierno del mes de mayo de 1921, era indispensable su "urgente habilitación". ${ }^{63}$

En el mientras tanto, los refugios provisorios, y sobre todo las instituciones particulares o las casas de familia encontradas por los defensores para colocar a los menores a su cargo, siguieron siendo los destinos para esa gran cantidad de niños, niñas y jóvenes que en toda la provincia requerían de una solución diferente tal como se pregonaba.

${ }^{61}$ Este decreto fue de la mano de una iniciativa originalmente presentada por el diputado Damonte antes comentada. Registro Oficial de la Provincia de Buenos Aires. Año 1918. T. 1, enero a junio. La Plata, Taller de Impresiones Oficiales, 1919. Departamento de gobierno. Decreto núm. 313. 11 de julio de 1918, p. 819. ALPBA, La Plata, Argentina.

${ }^{62}$ Registro Oficial de la Provincia de Buenos Aires. Año 1920. T. 1, enero a junio. La Plata, Taller de Impresiones Oficiales, 1924. Departamento de gobierno. Decreto núm. 159. 23 de marzo de 1920, pp. 253-254. ALPBA, La Plata, Argentina.

${ }^{63}$ Por ello, autorizaba a la Inspección General de Prisiones para instalar en el local que ya habían refaccionado la luz eléctrica. Registro Oficial de la Provincia de Buenos Aires. Año 1921. T. 1, enero a junio. La Plata, Taller de Impresiones Oficiales, 1924. Departamento de gobierno. Exp. Letra D, núm. 136. 2 de mayo de 1921, p. 360. En julio de ese mismo año autorizaron también un pago por instalaciones de baños y obras sanitarias. Registro Oficial de la Provincia de Buenos Aires. Año 1921. T. 2, julio a diciembre. La Plata, Taller de Impresiones Oficiales, 1924. Departamento de Gobierno. Exp. Letra P, núm. 425. 11 de julio de 1921, p. 836. ALPBA, La Plata, Argentina. 


\section{A MODO DE CIERRE}

En abril de 1921, en medio de presiones políticas imposibles de seguir ignorando, acorralado ante la posibilidad de una intervención federal y sin los apoyos necesarios en la legislatura donde muchos de los miembros de su propio partido respondían a la facción opositora del mismo, Crotto presentó la renuncia a la gobernación. El vicegobernador Luis Monteverde se hizo cargo de la primera magistratura hasta completar el mandato.

Para entonces, el Patronato seguía siendo un proyecto que para realizarse de manera acabada necesitaba, como punto de partida, el edificio tal como se había concebido en 1910. Esto era fundamental tanto para dar cabida a todos los menores de ambos sexos que deberían quedar comprendidos bajo su ala de protección, así como a la instalación de talleres para la formación en oficios que era, como señalamos, una parte esencial del proyecto.

A pesar de que la prensa opositora e independiente fustigó incansablemente en relación con el Patronato y a la poca acción del poder ejecutivo, lo cierto es que el radicalismo tomó una serie de medidas para poner a andar un proyecto que le precedía. El interventor reglamentó el refugio de la calle 6 que Ugarte había destinado de manera provisoria, y Crotto se preocupó por las menores de sexo femenino y por dar inicio a la obra del edificio que permitiría por fin hacer realidad el anhelado proyecto de asilo y reforma de menores.

¿Alcanza esto para señalar que el arribo del radicalismo, embanderado tras el respeto a las instituciones políticas fue un parteaguas en materia de política social por la infancia? Entiendo que no.

Tanto Cantilo en su breve estadía en la primera magistratura, pero especialmente Crotto después, mostraron al respecto un reformismo moderado, que los llevó a retomar proyectos que aunque no habían concebido ellos mismos, se basaban en diseños y concepciones con los que consensuaban y que en esencia no buscaron modificar en forma sustancial.

El radicalismo en muchos aspectos fue protagonista de un nuevo tiempo político en el que, entre otras cosas, se llegaba al poder por el voto que la ley de 1912 consagró. Como sostiene Hora (2013), aunque muchas de las viejas prácticas políticas como la violencia o el clientelismo basado en la utilización discrecional de los recursos públicos no desparecieron, no es menos cierto que al electorado había ahora que interpelarlo desde un lugar diferente: había que convencerlo para obtener buenos resultados en las urnas, y este partido supo interpretar bien las corrientes de opinión predominantes en esa etapa. 
La infancia y el problema que representaba en un país pretendidamente moderno, era parte de viejos reclamos que intelectuales, funcionarios, legisladores y prensa de las más variadas orientaciones políticas manifestaban desde hacía más de una década. Sostengo así que la realidad que la infancia desamparada suponía, en un marco de crisis económica, escasez laboral y agitación social sumada a la enorme presión que la opinión pública ejerció sobre el tema, antes que un convencimiento particular sobre el mismo, llevó al primer gobierno radical a tomar medidas para hacer realidad el proyecto pensado por quienes le precedieron en el poder. Sin embargo, y más allá de algunos discursos particulares, encendidos e insistentes, una agenda en la que los menores no parecieron ocupar el primer lugar en las prioridades del gobernador Crotto, lo llevó a tomar el tema con cierta moderación y mucha demora. Esa mesura no le permitió hacerlo ni con la urgencia reclamada ni con la magnitud necesaria para hacer realidad lo que hasta entonces seguía siendo una meta a alcanzar: resolver por fin el problema de la infancia pobre, abandonada y mendicante de la provincia de Buenos Aires.

\section{LISTA DE REFERENCIAS}

Ascolani, A. (2013). Trabajadores y sindicalismo. En J. M. Palacio (comp.), Historia de la Provincia de Buenos Aires. T. 4 (pp. 393-417). Buenos Aires: Edhasa.

Aversa, M. M. (2015). Un mundo de gente menuda. El trabajo infantil tutelado, ciudad de Buenos Aires, 1870-1920. (Tesis de doctorado inédita). Facultad de Filosofía y Letras-Universidad de Buenos Aires, Argentina.

Barba, F. (2007). El proceso electoral de 1917 en la provincia de Buenos Aires y el triunfo de la UCR. Anuario del Instituto de Historia Argentina, 7, 11-29.

Barba, F. (2009). Un aspecto de la oposición radical yrigoyenista al gobernador Crotto, las interpelaciones parlamentarias. Anuario del Instituto de Historia Argentina, 9, 85-96.

Barba, F. (2010). José Camilo Crotto. Un gobierno en medio de la borrasca. La Plata: Instituto Cultural de la provincia de Buenos Aires/Archivo Histórico Dr. Ricardo Levene.

Béjar, M. D. (2002). Los conservadores bonaerenses: un partido desde el gobierno. Estudios Sociales, 22-23, 95-123.

Castro, M. (2000). Partidos políticos, opinión pública y estrategias de comunicación en los periodos pre-electorales. La provincia de Buenos Aires 1912-1941. En M. E. Spine- 
lli, A. Servetto, M. Ferrari y G. Closa (comps.), La conformación de las identidades políticas en la Argentina del siglo xx (pp. 125-156). Mar del Plata: Universidad Nacional de Córdoba/Universidad Nacional del Centro/Universidad Nacional de Mar del Plata.

Cerdá, J. M. (2013). Una política social particular para la infancia en la década del '30: el Patronato y los tribunales de menores en la provincia de Mendoza. Revista Páginas, 5(8), 197-220. Recuperado de http://revistapaginas.unr.edu.ar/index.php/ RevPaginas/article/view/74

De Paz Trueba, Y. (2014). El trabajo infantil en el centro y sur de la provincia de Buenos Aires. Niñas y niños a fines del siglo diecinueve y principios del veinte. Mundos do Trabalho, 6(12), 177-195. DoI: https://doi.org/10.5007/1984-9222.2014v6n12p177

De Paz Trueba, Y. (2017). Asilos, infancia y trabajo: la campaña bonaerense a fines del siglo xIX y comienzos del xx. Andes, 1(28), 1-14.

De Paz Trueba, Y. (2018a). Discursos y prácticas políticas hacia la infancia en la provincia de Buenos Aires. Niñas y niños a principios del siglo xx. Revista de Indias, 78(272), 237-261. DoI: https://doi.org/10.3989/revindias.2018.008

De Paz Trueba, Y. (2018b). El Patronato Provincial de Menores: idas y vueltas de una iniciativa frustrada. Provincia de Buenos Aires, 1910-1916. Ponencia presentada en la XII Jornadas de Investigadores en Historia, Departamento de Historia Facultad de Humanidades-Universidad Nacional de Mar del Plata, Mar del Plata, Argentina.

Fernández Irusta, P. (2006). El Partido Conservador de la provincia de Buenos Aires y el proceso de democratización bonaerense, 1908-1918. Estudios Sociales, 31(1), 95135. DoI: https://doi.org/10.14409/es.v31i1.2582

Fernández Irusta, P. (2009). Los conservadores bonaerenses y la reforma de la ley electoral provincial (1910-1913). Boletín del Instituto Ravignani, 31, 79-122.

Ferrari, M. (2008). Los políticos en la república radical. Prácticas políticas y construcción de poder. Buenos Aires: Siglo XXI.

Ferrari, M. (2012). De la nación a las provincias. Adaptaciones de la Ley Sáenz Peña. Estudios Sociales, 43(1), 183-204. Dor: https://doi.org/10.14409/es.v43i1.2708

Fuentes, L. (2016). Conservadores y radicales en el interior bonaerense. Ayacucho, Azul, Lobería y Tandil, 1910-1943. (Tesis de doctorado inédita). Facultad de Ciencias Humanas-Universidad Nacional del Centro de la Provincia de Buenos Aires, Tandil, Argentina.

Hora, R. (2013). La política bonaerense: del orden oligárquico al imperio del fraude. En J. M. Palacio (comp.), Historia de la Provincia de Buenos Aires. T. 4 (pp. 51-80). Buenos Aires: Edhasa.

Melon Pirro, J. (1994). La ley Sáenz Peña de Ugarte, o el éxito de la reforma conservadora en la Provincia de Buenos Aires. En F. Devoto y M. Ferrari (comps.), La 
construcción de las democracias rioplatenses: proyectos institucionales y prácticas políticas, 1900-1930 (pp.107-135). Buenos Aires: Biblos.

Moreyra, B. (2009). Cuestión social y políticas sociales en la Argentina. La modernidad periférica, Córdoba 1900-1930. Buenos Aires: UNQUI.

Palacio, J. M. (2000). La antesala de lo peor: la economía argentina entre 1914 y 1930. En R. Falcón (dir.), Nueva historia argentina. T. vi. Democracia, conflicto social y renovación de ideas, 1916-1930 (pp. 101-150). Buenos Aires: Sudamericana.

Persello, A. V. (2004). El Partido Radical. Gobierno y oposición, 1916-1943. Buenos Aires: Siglo XXI.

Remedi, F. (2019). Miradas sobre la mendicidad infantil en la ciudad de Córdoba en las primeras décadas del siglo xx. Páginas, 27. Recuperado de http://revistapaginas.unr.edu.ar/index.php/RevPaginas

Rocchi, F. (2013). La economía bonaerense: del auge exportador a su crisis. En J. M. Palacio (comp.), Historia de la Provincia de Buenos Aires. T. 4 (pp. 81-121). Buenos Aires: Edhasa.

Salvatore, R. (2016). Burocracias expertas y exitosas en Argentina: los casos de educación primaria y salud pública (1870-1930). Estudios Sociales del Estado, 2(3), 22-64. Recuperado de http://estudiossocialesdelestado.org/index.php/ese/article/view/75

Sosensky, S. (2008). Un remedio contra la delincuencia: el trabajo infantil en las instituciones de encierro de la ciudad de México durante la posrevolución. Asclepio. Revista de Historia de la Medicina y de la Ciencia, Lx(2), 95-118. DoI: https://doi. org/10.3989/asclepio.2008.v60.i2.259

Stagno, L. (2008). Cuando la educación debió sustituir al castigo. Sobre las instituciones educativas ligadas al complejo tutelar, Bs. As., 1919-1942. Ponencia presentada en la IV Jornada Argentina de Historia de la Educación, Salta, Argentina.

Stagno, L. (2010). Una infancia aparte. La minoridad en la provincia de Buenos Aires. Buenos Aires: Flacso/Libros Libres.

Tato, M. I. (2005). Variaciones reformistas: los conservadores bonaerenses ante el desafío de la democratización, 1912-1919. Secuencia, 63, 128-150. Dor: http://dx.doi. org/10.18234/secuencia.v0i63.933

Zapiola, C. (2014). Un lugar para los menores. Patronato estatal e instituciones de corrección. Buenos Aires, 1890-1930. (Tesis de doctorado inédita). Facultad de Filosofía y Letras-Universidad de Buenos Aires, Argentina.

Zapiola, C. (2009). Los niños entre la escuela, el taller y la calle (o los límites de la obligatoriedad escolar) Buenos Aires, 1884-1915. Cadernos de Pesquisa, 39(136), 6991. DoI: http://dx.doi.org/10.1590/S0100-15742009000100005 